\title{
THE KOSZUL PROPERTY IN AFFINE SEMIGROUP RINGS
}

\author{
JÜrgen Herzog, Vic Reiner AND Volkmar Welker
}

\begin{abstract}
We investigate the Koszul property for quotients of affine semigroup rings by semigroup ideals. Using a combinatorial and topological interpretation for the Koszul property in this context, we recover known results asserting that certain of these rings are Koszul. In the process, we prove a stronger fact, suggesting a more general definition of Koszul rings, already considered by Fröberg. This more general definition of Koszulness turns out to be satisfied by all Cohen-Macaulay rings of minimal multiplicity.
\end{abstract}

\section{Introduction.}

The main point in this paper is an interplay between topological combinatorics and commutative algebra. Our goal is to relate the homological properties of certain rings to the topology of certain poset complexes.

We begin by describing the rings to be studied. Let $\Lambda$ be an associative commutative finitely generated semigroup with a neutral element and satisfying the cancellation rule: $\lambda_{1} \lambda=\lambda_{2} \lambda$ implies $\lambda_{1}=\lambda_{2}$. We further assume that no element of $\Lambda$ is invertible. We call such a semigroup affine since it may be identified with a semigroup generated by a finite set of monomials in a polynomial ring, or, in additive notation, as a sub-semigroup of $\mathbf{N}^{d}$ for some $d$. We can write the elements of an affine semigroup $\Lambda$ in either additive or multiplicative notation, once we identify an element $\lambda=\left(\lambda_{1}, \ldots, \lambda_{d}\right) \in \mathbf{N}^{d}$ with the monomial $\mathbf{z}^{\lambda}=z_{1}^{\lambda_{1}} \cdots z_{d}^{\lambda_{d}}$. Addition of vectors corresponds to multiplication of monomials and we write 0 or 1 for the neutral element of $\Lambda$. Consider the semigroup ring $k[\Lambda]$ of $\Lambda$ over a field $k$. The toric ideal $I_{\Lambda}$ is the kernel of the map $\phi: k\left[x_{1}, \ldots, x_{n}\right] \rightarrow k\left[z_{1}, \ldots, z_{d}\right]$ sending $x_{i}$ to $\mathbf{z}^{\alpha_{i}}:=z_{1}^{\alpha_{i 1}} z_{2}^{\alpha_{i 2}} \cdots z_{d}^{\alpha_{i d}}$, where the $\alpha_{i}$ 's are minimal generators of the semigroup. Consequently, we have

$$
k\left[x_{1}, \ldots, x_{n}\right] / I_{\Lambda} \cong k[\Lambda] \subset k\left[z_{1}, \ldots, z_{d}\right] .
$$

Let $J$ be an ideal in $k[\Lambda]$ that is generated by semigroup elements, i.e. a semigroup ideal. We denote by $R$ the quotient ring $k[\Lambda] / J$. 
In the spirit of $[\mathbf{B H 2}]$ we extend a result in $[\mathbf{L S}],[\mathrm{PRS}]$ showing that the computation of $\operatorname{Tor}^{R}(k, k)$ is equivalent to the computation of the relative homology of order complexes of the divisor poset of the semigroup and certain combinatorially defined sub-complexes (Theorem 2.1). Section 3 gives a more concrete geometric description (Theorem 3.1) and topological analysis (Lemma 3.3) of these complexes whose proofs use results from the theory of subspace-arrangements and homotopy colimits as in $[\mathbf{W Z Z}],[\mathbf{Z Z}]$.

In Section 4 we apply this topological information to prove that $R$ is Koszul (see definition in Section 2) in the cases when either $\Lambda=\mathbf{N}^{d}$ and $J$ is a quadratic monomial ideal (Corollary 4.1), or $J=0$ and $\Lambda$ has minimal generators $\alpha_{1}, \ldots, \alpha_{n}$ for which the toric ideal $I_{\Lambda}$ has a quadratic Gröbner basis (Corollary 4.3). In the first case, $R$ was shown to be Koszul by Fröberg [Fr1], who explicitly described the generalized Koszul complex providing the linear minimal free resolution of $k$. In the second case, if $I_{\Lambda}$ is graded with respect to the grading with $\operatorname{deg}\left(x_{i}\right)=1$ then $R$ is again known to be Koszul; this can be proved using the first case along with standard deformation arguments (cf. [BHV] or [Ei, Section 15]). However our topological techniques also apply when $I_{\Lambda}$ is not graded in the above way, and in particular Theorem 4.2 generalizes some of the results in [PRS] which used the technique of non-pure shellability. In particular, we recover the results of [PRS] giving a rational expression for the Poincaré series of $\operatorname{Tor}^{R}(k, k)$ when $R$ is any 2-dimensional normal affine semigroup ring.

Furthermore, Corollary 4.3 inspired the developments of Section 5, in which we generalize the notion of Koszul algebras to local rings and to $\mathbf{N}^{d}$ graded $k$-algebras. We call such a ring Koszul if its associated graded ring is Koszul in the ordinary sense. This notion is justified by the fact, first proved by Fröberg [Fr2], that in this case the Poincaré series of the ring and its associated graded ring coincide. Along with providing an algebraic proof of Corollary 4.3, we also prove (Theorem 5.2) that this new notion of Koszulness applies to Cohen-Macaulay rings of minimal multiplicity. As a consequence we obtain a rational expression for the Poincaré series of such rings, generalizing the case of 2-dimensional normal affine semigroup rings.

\section{Computing Betti numbers of rings by simplicial complexes.}

Computation of Betti numbers of rings using relative homology was introduced by Bruns and Herzog in [BH2] for finite resolutions of monomial ideals in semigroup rings. The main result of this section, Theorem 2.1, is in the same spirit, but deals with infinite resolutions. In the case when $J=0$ the formula in Theorem 2.1 was proved in $[\mathbf{L S}]$ and $[\mathbf{P R S}]$. The idea is to identify the chains in the Bar resolution of $k$ with the chains of certain 
simplicial complexes, as we now discuss.

The $k$-algebra $R$ is naturally augmented with augmentation ideal $\mathfrak{m}=$ $\bigoplus k \lambda$. Note that $R$ is a $\Lambda$-graded $k$-algebra, and $\mathfrak{m}$ a $\Lambda$-graded $R$ $\lambda \in \Lambda, \lambda \neq 0$

module. Indeed, we may define $R_{\lambda}=k \lambda$ for all $\lambda \in \Lambda$, and similarly for $\mathfrak{m}$. Thus the minimal free resolution of $k$ over $R$ and the Tor-groups $\operatorname{Tor}_{i}^{R}(k, k)$ are naturally $\Lambda$-graded $k$-vector spaces, and we will denote by $\operatorname{Tor}_{i}^{R}(k, k)_{\lambda}$ the $\lambda$-graded piece of $\operatorname{Tor}_{i}^{R}(k, k)$.

In order to compute the Tor-groups we must construct certain simplicial complexes. First, partially order the semigroup $\Lambda$ by divisibility: For $\lambda, \mu \in$ $\Lambda$, say $\mu$ divides $\lambda$ (written $\mu \leq \lambda$ ) if $\lambda=\mu+\iota$ for some $\iota \in \Lambda$ Then the closed and open intervals

$$
[\lambda, \mu]:=\{\rho \in \Lambda: \mu \leq \rho \leq \lambda\}, \quad(\lambda, \mu):=\{\rho \in \Lambda: \mu<\rho<\lambda\}
$$

are partially ordered sets with the induced ordering.

Associated with a poset $P$ is its order complex $\Delta(P)$. The ground set of $\Delta(P)$ is the set of elements of $P$, and faces are the sub-sets $\left\{p_{1}, \ldots, p_{k}\right\}$ of $P$ whose elements form a chain $p_{1}<p_{2}<\cdots<p_{k}$. Define $\Delta_{\lambda}:=\Delta((0, \lambda))$, i.e. $\Delta_{\lambda}$ is the order complex for the open interval lying strictly between 0 and $\lambda$ in the poset structure on $\Lambda$. Note that the complexes $\Delta_{\lambda}$ are not pure (all maximal faces of the same dimension) unless the minimal generators of the monoid $\Lambda$ lie on a hyperplane in $\mathbf{N}^{d}$.

Let $J$ be an ideal in $k[\Lambda]$ that is generated by semigroup elements, what we call a semigroup ideal. We will often abuse notation and use $J$ both to denote the ideal in the ring, and to denote the set $\left\{\lambda \in \Lambda: \mathbf{z}^{\lambda} \in J\right\}$. In the case $\Lambda=\mathbf{N}^{d}$ the ideal $J$ is called a monomial ideal. For $\lambda \in \Lambda$ we denote by $\Delta_{\lambda, J}$ the sub-complex of $\Delta_{\lambda}$ consisting of all chains $\lambda_{1}<\cdots<\lambda_{l}$ in $(0, \lambda)$ such that if we set $\lambda_{0}=0$ and $\lambda_{l+1}=\lambda$ then there is an index $0 \leq i \leq l$ such that

$$
\lambda_{i+1}-\lambda_{i} \in J
$$

In other words, $\Delta_{\lambda, J}$ is the sub-set of $\Delta_{\lambda}$ consisting of the chains which contain a "jump" from $J$. Since $J$ is an ideal, this sub-set is actually a simplicial sub-complex of $\Delta_{\lambda}$. The (relative, reduced) simplicial homology of the pair $\left(\Delta_{\lambda}, \Delta_{\lambda, J}\right)$ with coefficients in $k$ will be denoted by $\tilde{\mathrm{H}}_{i}\left(\Delta_{\lambda}, \Delta_{\lambda, J} ; k\right)$. Furthermore, we denote by $\tilde{C} .\left(\Delta_{\lambda}, \Delta_{\lambda, J}\right)$ the augmented oriented chain complex of the pair $\left(\Delta_{\lambda}, \Delta_{\lambda, J}\right)$.

Theorem 2.1. Let $\Lambda \subseteq \mathbf{N}^{d}$ be an affine semigroup and let $J$ be a semigroup ideal in $k[\Lambda]$. Set $R=k[\Lambda] / J$. Then

$$
\operatorname{Tor}_{i}^{R}(k, k)_{\lambda} \cong \tilde{\mathrm{H}}_{i-2}\left(\Delta_{\lambda}, \Delta_{\lambda, J} ; k\right)
$$


for all $\lambda \in \Lambda$ and all $i>0$.

Proof. Consider the bar resolution B of $k$ over $k[\Lambda]$, see [Ma, Ch. 10,§2],

$$
\mathbf{B}: \cdots \rightarrow B_{i} \rightarrow B_{i-1} \rightarrow \cdots \rightarrow B_{0} \rightarrow k \rightarrow 0 .
$$

This is a particular free resolution of $k$ as an $R$-module, which is far from being minimal. In our case, $B_{0}=R$ and, for $i>0, B_{i}$ is the free $R$-module with basis $\left\{\left[\lambda_{1}|\cdots| \lambda_{i}\right]\right\}$, indexed by ordered $i$-tuples of non-zero elements of $\Lambda \backslash J$. The differential $d_{i}: B_{i} \rightarrow B_{i-1}$ acts by the rule

$$
d_{i}\left[\lambda_{1}|\cdots| \lambda_{i}\right]=z^{\lambda_{1}}\left[\lambda_{2}|\cdots| \lambda_{i}\right]+\sum_{1 \leq j \leq i-1}(-1)^{j} \cdot\left[\lambda_{1}|\cdots| \lambda_{j}+\lambda_{j+1}|\cdots| \lambda_{i}\right]
$$

in which $\left[\lambda_{1}|\cdots| \lambda_{i}\right]$ is interpreted as 0 whenever any $\lambda_{j}$ is in $J$. To compute $\operatorname{Tor}_{i}^{R}(k, k)$, we must tensor this resolution with the trivial $R$-module $k(=$ $R / \mathfrak{m})$. This yields the complex of $k$-vector spaces

$$
k \otimes \mathbf{B}: \cdots \rightarrow k \otimes B_{i} \rightarrow k \otimes B_{i-1} \rightarrow \cdots \rightarrow k \otimes B_{0}=k
$$

with differential $d_{i}\left[\lambda_{1}|\cdots| \lambda_{i}\right]=\sum_{1 \leq j \leq i-1}(-1)^{j} \cdot\left[\lambda_{1}|\cdots| \lambda_{j}+\lambda_{j+1}|\cdots| \lambda_{i}\right]$.

By definition, $\operatorname{Tor}_{i}^{R}(k, k)$ is the homology of this complex. Note that the differential preserves the sum $\lambda=\lambda_{1}+\cdots+\lambda_{i}$ of the entries in each bracket, that is, the Bar complex is the direct sum of its finite-dimensional graded components $(k \otimes \mathbf{B})_{\lambda}$. If we identify $\left[\lambda_{1}\left|\lambda_{2}\right| \cdots \mid \lambda_{i}\right]$ with the chain $\lambda_{1}<\lambda_{1}+\lambda_{2}<\cdots<\lambda_{1}+\cdots+\lambda_{i-1}$ in the open interval $(0, \lambda)$, then the differential of the Bar complex $(k \otimes \mathbf{B})_{\lambda}$ is precisely the boundary map in the augmented relative chain complex $\tilde{C}_{{ }_{-2}}\left(\Delta_{\lambda}, \Delta_{\lambda, J}\right)$.

Before closing this section, we give a brief discussion of Koszul rings in this context. When the generators of $\Lambda$ all lie on some hyperplane in $\mathbf{N}^{d}$, the toric ideal $I_{\Lambda}$ is homogeneous with respect to the $\mathbf{N}$-grading defined by $\operatorname{deg}\left(x_{i}\right)=1$ for all $i$, and $R$ and $\operatorname{Tor}^{R}(k, k)$ inherit this $\mathbf{N}$-grading. For any $\mathbf{N}$-graded $k$ algebra $R$, we let $\operatorname{Tor}_{i}^{R}(k, k)_{j}$ denote the $j^{\text {th }}$-graded component of $\operatorname{Tor}_{i}^{R}(k, k)$ with respect to this grading, and it is easy to see that $\operatorname{Tor}_{i}^{R}(k, k)_{j}=0$ for $j<i$. We say that $R$ is Koszul if $\operatorname{Tor}_{i}^{R}(k, k)_{j}=0$ for all $j \neq i$. Recall that $\operatorname{dim}_{k} \operatorname{Tor}_{i}^{R}(k, k)_{j}$ equals the number of basis elements of degree $j$ in the $i^{t h}$ free $R$-module occurring in a minimal resolution of $k$ as a trivial $R$-module. Because of this interpretation, Koszulness of $R$ is equivalent to this resolution being linear, i.e. all matrix entries for the boundary maps are either 0 or of degree 1 . Koszulness has important consequences for $R$ (see for example, Lemma 5.5), and in [PRS, Corollary 2.2] it was observed that Theorem 2.1. has the following consequence: In the case $J=\mathfrak{o}, R$ is Koszul if and only if $\Lambda$ is a Cohen-Macaulay poset (see [BGS] for information on Cohen-Macaulay posets). 
It follows from work of Fröberg [Fr1] that when $\Lambda=\mathbf{N}^{d}, R$ is Koszul if and only if the monomial ideal $J$ is generated by quadratic monomials. At present, we know of no example where $J=0$ and $I_{\Lambda}$ is generated by quadrics such that $R$ is not Koszul. On the other hand, the following example shows that a non-Koszul ring $R$ which is a quotient of quadrics can appear in our set-up, if we allow $J \neq 0$.

Example 2.2. Let $\Lambda$ be the monoid generated by $x=(0,2), y=(1,1)$, $z=(2,0)$ in $\mathbf{N}^{2}$. Taking the quotient by the semigroup ideal $J=\left\langle x y, z^{2}\right\rangle$ and the toric ideal $I_{\Lambda}=\left\langle y^{2}-x z\right\rangle$ we obtain the $\operatorname{ring} R=k[x, y, z] /\left\langle y^{2}-\right.$ $\left.x z, x y, z^{2}\right\rangle$. Consider the multidegree $\lambda=(4,4)$, which lies in degree 4 of the $\mathbf{N}$-grading. One can compute, for example with the computer algebra package MACAULAY, that $\operatorname{Tor}_{3}^{R}(k, k)_{(4,4)} \cong \tilde{\mathrm{H}}_{1}\left(\Delta_{(4,4)}, \Delta_{(4,4),\left\langle x y, z^{2}\right\rangle} ; k\right)$ does not vanish for any field $k$, so that $\operatorname{Tor}_{3}^{R}(k, k)_{4} \neq 0$ and $R$ is not Koszul. By $[\mathbf{B F}]$, up to an isomorphism, this is the only example of a non-Koszul ideal generated by quadratic binomials in three variables.

We close this section with the following simple consequence of Theorem 2.1 .

Proposition 2.3. Let $R$ be a quotient of an affine semigroup ring $k[\Lambda]$ by a semigroup ideal $J$. Then the minimal free resolution $(F ., d$.) of $k$ over $R$ is monomial, meaning that for $p \geq 0$ we can choose basis for $F_{p}$ so that the matrix for the differential $d_{p}: F_{p} \rightarrow F_{p-1}$ has monomial entries.

Proof. We know that the basis elements for the modules $F_{p}$ can be chosen to be homogeneous with respect to the $\Lambda$-grading, and also the maps can be chosen to preserve this $\Lambda$-grading. But this then implies that each entry in the map is a monomial, since the ring $R=k[\Lambda] / J$ has (up to multiplication by constants) a unique element in each multidegree and this element is represented by a monomial.

\section{Topological analysis.}

The goal of this section is to provide a more concrete geometric understanding of the pair $\left(\Delta_{\lambda}, \Delta_{\lambda, J}\right)$ in the case where $\Lambda=\mathbf{N}^{d}$. In this case we have $d=n$ and $k[\Lambda]=k\left[x_{1}, \ldots, x_{n}\right]$, so that $J \subseteq k\left[x_{1}, \ldots, x_{n}\right]$ is a monomial ideal, and an element $\lambda \in \Lambda$ is simply a monomial $m$ in $k\left[x_{1}, \ldots, x_{n}\right]$. We will present a detailed topological analysis of the complexes $\Delta_{m}$ and $\Delta_{m, J}$ in terms of subspace arrangements.

An arrangement of subspaces $\mathcal{A}$ in $\mathbf{R}^{n}$ is a finite collection of linear subspaces. We identify $\mathcal{A}$ with its union $\bigcup_{H \in \mathcal{A}} H$. Here we consider the real 
$n$-space $\mathbf{R}^{n}$ with coordinates given by $u_{1}, \ldots, u_{n}$ (since we are already using the natural variables $x_{1}, \ldots, x_{n}$ as polynomial ring variables).

Let $J \subset k\left[x_{1}, \ldots, x_{n}\right]$ be a monomial ideal, and among its minimal generators, let the square-free monomials be $m_{1}, \ldots, m_{l}$. Given a monomial $m$, let $U_{m}$ denote the subspace which is the intersection of all hyperplanes $u_{p}=u_{q}$ for monomials $x_{p} x_{q}$ dividing $m$. Then the canonical arrangement associated to $J$ is the subspace arrangement inside the hyperplane $u_{1}+\cdots+u_{n}=0$ consisting of its intersection with the union of all linear subspaces $U_{m_{i}}$. For example, if $J=\left(x_{1}^{2} x_{2}, x_{1} x_{3}, x_{2}^{4}, x_{2} x_{3} x_{4}\right) \subset k\left[x_{1}, x_{2}, x_{3}, x_{4}\right]$, then $\mathcal{A}_{J}$ is the union of the subspaces $\left\{u_{1}=u_{3}\right\} \cup\left\{u_{2}=u_{3}=u_{4}\right\}$ inside the hyperplane $u_{1}+u_{2}+u_{3}+u_{4}=0$.

Theorem 3.1. Let $J$ be a monomial ideal and $m$ a square-free monomial in $k\left[x_{1}, \ldots, x_{n}\right]$, say without loss of generality, $m=x_{1} \cdots x_{e}$. Then the pair $\left(\Delta_{m}, \Delta_{m, J}\right)$ is homeomorphic to the pair $\left(\mathbf{S}^{e-2}, \mathbf{S}^{e-2} \cap \mathcal{A}_{J^{e}}\right)$, where $\mathbf{S}^{e-2}$ is the unit sphere in the hyperplane $u_{1}+\cdots+u_{e}=0$ in real e-space and $\mathcal{A}_{J^{e}}$ is the canonical arrangement corresponding to $J^{e}=J \cap k\left[x_{1}, \ldots, x_{e}\right]$ in $\mathbf{R}^{e}$.

Proof. The proof consists of identifying particular geometric realizations of $\Delta_{m}$ and $\Delta_{m, J}$. The complex $\Delta_{m}$ is easily seen to be the order complex of the proper part of the Boolean lattice $B_{e}$ on an $e$-element set, i.e. it is the simplicial complex of chains of proper sub-sets of an $e$-element set. To see this, identify a monomial with the set of indices of the occurring variables. Thus $\Delta_{m}$ is the simplicial complex associated to the barycentric subdivision of the boundary of an $(e-1)$-simplex (sometimes known as the Coxeter complex for type $A_{e-1}$ ). In particular, $\Delta_{m}$ is homeomorphic to $\mathbf{S}^{e-2}$. If we embed this $(e-1)$-simplex so that its vertices are the standard basis vectors in $\mathbf{R}^{e}$, then it will naturally lie in the affine hyperplane $u_{1}+\cdots+u_{e}=1$. A face of $\Delta_{m}$ corresponds to a chain of sub-sets

$$
\emptyset=A_{0} \subset A_{1} \subset \cdots \subset A_{r} \subset A_{r+1}=\{1,2 \ldots, e\} .
$$

One can check that geometrically the relative interior of this face is the set of points in the affine hyperplane $u_{1}+\cdots+u_{e}=1$ satisfying the conditions (a) $u_{i}=u_{i^{\prime}}$ if $i, i^{\prime}$ lie together in some set $A_{j+1}-A_{j}$, and (b) $u_{i}<u_{i^{\prime}}$ if $i \in A_{j}$ and $i^{\prime} \in A_{j^{\prime}}$ for some $j<j^{\prime}$.

We obtain a homeomorphic picture if we first translate the entire $(e-$ $1)$-simplex by the vector $\frac{-1}{e}(1, \ldots, 1)$ so that the simplex now lies in the hyperplane $u_{1}+\cdots+u_{e}=0$, and then map each point of the boundary of the simplex radially to the point on the unit sphere $\mathbf{S}^{e-2}$ in $u_{1}+\cdots+u_{e}=0$ which lies on the same ray from the origin. Now the relative interior of the above face is the set of points on the unit sphere satisfying the same 
conditions (a) and (b) above. The advantage to this situation is that now every subspace of the form $u_{i_{1}}=\cdots=u_{i_{r}}$ has its intersection with the sphere $\mathbf{S}^{e-2}$ triangulated as a sub-complex of the Coxeter complex $\Delta_{m}$.

By the definition of $\Delta_{m, J}$, it will be the sub-complex consisting of all such chains in which there is an index $j$ for which $\prod_{i \in A_{j+1}-A_{j}} x_{i}$ is divisible by a square-free monomial in $J$. Comparing this with the description in (a) and (b) above, these are exactly the faces in the barycentric subdivision which triangulate the intersection of the canonical arrangement $\mathcal{A}_{J^{e}}$ and the unit sphere $\mathbf{S}^{e-2}$.

The next two results give a closer topological analysis of the pair $\left(\Delta_{m}, \Delta_{m, J}\right)$ in the case where $J$ is a quadratic monomial ideal. To state these results, we need to establish some notation.

Say that a non-commutative monomial in $k\left\langle y_{1}, \ldots, y_{n}\right\rangle$ has content $m$ (or that it lies in the non-commutative fiber of $m$ ) if it maps to $m$ under the map

$$
k\left\langle y_{1}, \ldots, y_{n}\right\rangle \rightarrow k\left[x_{1}, \ldots, x_{n}\right]
$$

defined by $y_{i} \mapsto x_{i}$. Here we use the notation $k\langle\cdots\rangle$ and $k[\cdots]$ to denote non-commutative and commutative polynomial rings, and all ideals in the non-commutative ring $k\left\langle y_{1}, \ldots, y_{n}\right\rangle$ are assumed to be two-sided. Given a quadratic monomial ideal $J$ in $k\left[x_{1}, \ldots, x_{n}\right]$, let its complementary ideal $J^{\prime}$ be the ideal generated by the set of all quadratic monomials in $k\left[x_{1}, \ldots, x_{n}\right]$ that do not lie in $J$. Given two non-commutative monomials, say that they are $J^{\prime}$-commuting equivalent if they have the same image under the quotient map

$$
k\left\langle y_{1}, \ldots, y_{d}\right\rangle \rightarrow k\left\langle y_{1}, \ldots, y_{d}\right\rangle /\left(y_{i} y_{j}-y_{j} y_{i}: x_{i} x_{j} \in J^{\prime}\right) .
$$

Lastly, say that a non-commutative monomial is $J^{\prime}$-non-stuttering if no rearrangement in its $J^{\prime}$-commuting class contains two adjacent letters $y_{i} y_{i}$ with $x_{i}^{2} \in J^{\prime}$. For a (commutative) monomial $m$ we denote by $\mathcal{C}(m)$ the set of $J^{\prime}$-non-stuttering $J^{\prime}$-commuting classes of non-commutative monomials with content $m$.

The next result further analyzes the case where $m$ is square-free and $J$ is quadratic. Of course, since $m$ is square-free all $J^{\prime}$-commuting classes are $J^{\prime}$-non-stuttering.

Lemma 3.2. Let $J, m$ be as in Theorem 3.1. If in addition $J$ is a quadratic monomial ideal then, $\mathcal{A}_{J^{e}}$ is an arrangement of hyperplanes, and the set of chambers cut out by $\mathcal{A}_{J}$ is in bijection with the set $\mathcal{C}(m)$.

Proof. When $J$ is a quadratic monomial ideal, it is clear from the definition that $\mathcal{A}_{J^{e}}$ is the arrangement of hyperplanes (inside $\sum_{i} u_{i}=0$ ) which is the 
union of all $u_{i}=u_{j}$ with $x_{i} x_{j} \in J$. So without loss of generality, it only remains to identify the chambers of $\mathcal{A}_{J}$ in case $J$ is an ideal generated by square-free quadratic monomials.

In this case, $\mathcal{A}_{J}$ is a sub-arrangement of the braid arrangement $\mathcal{A}_{e-1}$ which has all hyperplanes of the form $u_{i}=u_{j}$ with $1 \leq i<j \leq n$. Notice that the chambers cut out by the braid arrangement are of the form $u_{\sigma_{1}}>\cdots>$ $u_{\sigma_{e}}$ for some permutation $\sigma: i \mapsto \sigma_{i}$ of $\{1,2, \ldots, e\}$, corresponding to the maximal faces of the Coxeter complex $\Delta_{m}$. Note that these chambers also biject with the non-commutative monomials of content $m$. Since $\mathcal{A}_{J}$ is a subarrangement of $\mathcal{A}_{e-1}$, its chambers will be unions of chambers from $\mathcal{A}_{e-1}$, i.e. they will correspond to certain sub-sets of non-commutative monomials, which we must identify.

The key observation is that $\mathcal{A}_{J}$ is a graphic arrangement [OT, §2.4]: To a graph $G$ (with no loops or multiple edges) on $e$ vertices one associates the arrangement $\mathcal{A}_{G} \subseteq \mathcal{A}_{e-1}$ consisting of all hyperplanes $u_{i}=u_{j}$ for all edges $\{i, j\}$ in $G$. In particular, $\mathcal{A}_{e-1}=\mathcal{A}_{K_{e}}$ for $K_{e}$ the complete graph on $e$ vertices. The graphic arrangements $\mathcal{A}_{G}$ are exactly the arrangements $\mathcal{A}_{J}$ for ideals $J$ generated by square-free quadratic monomials. It is known [GZ] that the chambers of a graphic arrangement $\mathcal{A}_{G}$ are in bijection with the acyclic orientations of the edges of $G$ (i.e., the directed graphs containing no directed cycles which are obtained by picking a direction for each of $G$ ). One can also view such an acyclic orientation $\Omega$ as defining a poset structure $P_{\Omega}$ on the vertices of $G$, where $v>v^{\prime}$ in $P_{\Omega}$ if there is a directed edge from $v$ to $v^{\prime}$.

If $G$ is the graph associated to the square-free monomial ideal $J$, meaning $\{i, j\}$ is an edge of $G$ exactly when $x_{i} x_{j} \in J$, it is easy to see that these acyclic orientations biject to the set $\mathcal{C}(m)$ of all $J^{\prime}$-commuting equivalence classes of permutations $\omega$, under the correspondence which sends $\Omega$ to the set of all of linear extensions of the poset $P_{\Omega}$. The inverse correspondence which produces a poset $P$ (and hence an acyclic orientation) from a $J^{\prime}$-commuting equivalence class of permutations $\omega$ was first described by Cartier and Foata [CF] (see also [Sta, Exercise 3.48, p. 163]). Also note that in this case since $m$ is square-free no element of $\mathcal{C}(m)$ can $J^{\prime}$-stutter, so the set of $J^{\prime}$ commuting classes of permutations $\omega$ is the same as the set $\mathcal{C}(m)$. Therefore the chambers of $\mathcal{A}_{J}$ and the acyclic orientations of $G$ are both in bijection with $\mathcal{C}(m)$.

The next result analyzes the case where $m$ is not square-free.

Lemma 3.3. Let $J$ be a monomial ideal, and $m$ a monomial of degree $e$ in $k\left[x_{1}, \ldots, x_{n}\right]$. 
(1) If $m$ is not square-free then $\Delta_{m}$ is homeomorphic to a polytopal ball $\mathbf{B}^{e-2}$.

(2) If in addition $J$ is generated by quadratic monomials then the subcomplex $\Delta_{m, J}$ is homotopy equivalent to a one-point wedge of $(e-3)$ spheres indexed by $\mathcal{C}(m)$, i.e. to

$$
\bigvee_{\omega \in \mathcal{C}(m)} \mathbf{S}^{e-3}
$$

The proof of this lemma will occupy the remainder of this section.

Proof. Let $m=x_{1}^{e_{1}} \cdots x_{n}^{e_{n}}$ be a not necessarily square-free monomial of degree $e$. We polarize $m$ to obtain a square-free monomial $m^{\text {pol}}$ :

$$
m^{\mathrm{pol}}=x_{1}^{(1)} x_{1}^{(2)} \cdots x_{1}^{\left(e_{1}\right)} \cdots x_{n}^{(1)} x_{n}^{(2)} \cdots x_{n}^{\left(e_{n}\right)} .
$$

Consider the braid arrangement $A_{e-1}$ associated to $m^{\text {pol }}$ as in the proof of the previous lemma, lying in the space having coordinates

$$
u_{1}^{(1)}, u_{1}^{(2)}, \ldots, u_{1}^{\left(e_{1}\right)}, \ldots, u_{n}^{(1)}, u_{n}^{(2)}, \ldots, u_{n}^{\left(e_{n}\right)} .
$$

We claim that the simplicial complex $\Delta_{m}$ is then isomorphic to the subcomplex of the Coxeter complex $\Delta_{m \text { pol }}$ which covers the intersection of $\mathbf{S}^{e-2}$ (the unit sphere in the hyperplane $\sum_{i} u_{i}=0$ ) with the convex cone defined by

$$
\begin{gathered}
u_{1}^{(1)} \geq u_{1}^{(2)} \cdots \geq u_{1}^{\left(e_{1}\right)} \\
\vdots \\
u_{n}^{(1)} \geq u_{n}^{(2)} \cdots \geq u_{n}^{\left(e_{n}\right)} .
\end{gathered}
$$

To see this claim, recall that a maximal face in $\Delta_{m}$ corresponds to a noncommutative monomial $y_{i_{1}} \cdots y_{i_{e}}$ having content $m$. Replacing the $j^{\text {th }}$ occurrence of $y_{i}$ in the monomial by $y_{i}^{(j)}$, we get another non-commutative monomial $y_{i_{1}}^{\left(j_{1}\right)} \cdots y_{i_{e}}^{\left(j_{e}\right)}$, and this corresponds to the maximal face in $\Delta_{m^{\text {pol }}}$ lying inside the above cone and cut out by the stronger set of inequalities

$$
u_{i_{1}}^{\left(j_{1}\right)} \geq \cdots \geq u_{i_{e}}^{\left(j_{e}\right)} .
$$

As one varies over the non-commutative monomials of content $m$, these smaller cones cover the cone defined by (3.1), proving the claim.

The previous argumentation shows that $\Delta_{m}$ is homeomorphic to a ball $\mathbf{B}^{e-2}$. We can visualize this as a polytopal ball in the following way. If we 
assume (without loss of generality) that $e_{1} \geq 2$, then the radial projection of the intersection of the cone and sphere onto the affine hyperplane $u_{1}^{(1)}-$ $u_{1}^{(2)}=1$ is a homeomorphism of $\Delta_{m}$ onto the convex polytope defined by the inequalities (3.1) inside this affine hyperplane. Assertion (1) of Lemma 3.3 is now proved.

We move on to proving assertion (2), so from now on assume that $J$ is generated by quadratic monomials. We polarize the monomial ideal $J$ as follows. Consider the map

$$
k\left[x_{1}^{(1)}, x_{1}^{(2)}, \ldots, x_{1}^{\left(e_{1}\right)}, \ldots, x_{n}^{(1)}, x_{n}^{(2)}, \ldots, x_{n}^{\left(e_{n}\right)}\right] \rightarrow k\left[x_{1}, \ldots, x_{n}\right]
$$

which sends $x_{i}^{(j)}$ to $x_{i}$ for each $i$ and $j$, and let $J^{\text {pol }}$ be the ideal in the domain which is generated by all square-free monomials that map to a (quadratic) monomial which is a minimal generator of $J$ in $k\left[x_{1}, \ldots, x_{n}\right]$. It is clear that the sub-arrangement $\mathcal{A}_{J \text { pol }}$ of $\mathcal{A}_{e-1}$ gives a polytopal decomposition of the polytopal ball $\Delta_{m}$. Note however that some of the subspaces in $\mathcal{A}_{J^{\text {pol }}}$ may intersect only the boundary but not the interior of this ball. So these subspaces do not participate in the decomposition.

The maximal cells in this decomposition will correspond to chambers in $\Delta_{m^{\text {pol }}}$ lying in the convex cone described by the inequalities (3.1), and these chambers exactly correspond to the set of $J^{\prime}$-commuting equivalence classes $\omega$ of non-commutative monomials of content $m$, as in the proof of Lemma 3.2. For each such class $\omega$, let $\Delta_{\omega}$ denote the sub-complex of $\Delta_{m}$ which triangulates this chamber. We can then consider the covering $\mathcal{U}_{m}$ of $\Delta_{m}$ by the maximal cells $\Delta_{\omega}$ in the above polytopal decomposition. On the top of the intersection poset of this covering we add an artificial $\hat{1}$ (corresponding to the empty space).

Now consider the sub-complex $\Delta_{m, J}$, which we may identify with the arrangement $\mathcal{A}_{J^{\text {pol }}}$ intersected with the ball $\Delta_{m}$. For each maximal cell $\Delta_{\omega}$ in the polytopal decomposition of $\Delta_{m}$, let $\Delta_{\omega, J}$ denote the intersection $\Delta_{\omega} \cap \Delta_{m, J}$.

Clearly the $\left\{\Delta_{\omega, J}\right\}$ form a cover $\mathcal{U}_{m, J}$ of $\Delta_{m, J}$.

Lemma 3.4. In the covering $\mathcal{U}_{m}$ of $\Delta_{m}$ by the maximal cells $\left\{\Delta_{\omega}\right\}$, every proper intersection lies in the sub-complex $\Delta_{m, J}$.

Proof. Since the covering of $\Delta_{m}$ by these maximal cells was the polytopal decomposition cut out by the arrangement $\mathcal{A}_{J^{\mathrm{pol}}}$, all proper intersections of the maximal cells lie inside some hyperplane of this arrangement, and hence inside $\Delta_{m, J}$.

As a consequence of this lemma, the coverings $\mathcal{U}_{m}$ and $\mathcal{U}_{m, J}$ have isomorphic intersection posets, and differ only in the spaces assigned to minimal 
elements $p$ in the poset, where $\mathcal{U}_{m}$ has the spaces $\Delta_{\omega}$ and $\mathcal{U}_{m, J}$ has the spaces $\Delta_{\omega, J}$.

Recall that if $J$ is a quadratic monomial ideal in $k\left[x_{1}, \ldots, x_{e}\right]$ then $J^{\prime}$ is the complementary quadratic monomial ideal, generated by the complementary set of quadratic monomials to those in $J$.

Lemma 3.5. For any $J^{\prime}$-commuting class $\omega$ of non-commutative monomials with content $m$, the space $\Delta_{\omega, J}$ is either a sphere $\mathbf{S}^{e-3}$ or ball $\mathbf{B}^{e-3}$, depending upon whether $\omega$ is $J^{\prime}$-non-stuttering or $J^{\prime}$-stuttering.

Proof. Since $\Delta_{\omega, J}=\Delta_{\omega} \cap \Delta_{m, J}$, it is a sub-complex of the boundary of the polytope $\Delta_{\omega}$. It is not hard to see that the walls of the chamber $\Delta_{\omega}$ in the arrangement $\mathcal{A}_{J \mathrm{pol}}$ correspond to all possible

- adjacent pairs of repeated letters, or

- adjacent pairs of letters corresponding to a quadratic monomial in $J$ occurring among the non-commutative monomials belonging to the class $\omega$. Therefore, if $\omega$ is $J^{\prime}$-non-stuttering then every such wall corresponds to a pair of letters that comes from a quadratic monomial in $J$, so every wall lies in $\Delta_{\omega, J}$. This implies $\Delta_{\omega, J}$ is the entire boundary sphere $\mathbf{S}^{e-3}$ of the polytopal ball $\Delta_{\omega}$.

On the other hand, assume some word in $\omega$ stutters on some elements of $J^{\prime}$. Classify the walls of the polytopal ball $\Delta_{\omega}$ according to whether they lie in $\Delta_{\omega, J}$ or not. We claim that the walls which do not lie in $\Delta_{\omega, J}$ have non-empty common intersection $F$, which must therefore be some boundary face $F$ of $\Delta_{\omega}$. To see this claim, note that each such wall not in $\Delta_{\omega, J}$ corresponds to an occurrence of a pair of adjacent equal letters $y_{i} y_{i}$ with $x_{i}^{2}$ in $J^{\prime}$ in some representative of the class $\omega$. It is not hard to see (and follows easily from Cartier-Foata theory $[\mathbf{C F}]$ ) that there is some representative of $\omega$ which has all possible such pairs of $J^{\prime}$-stutterings, and then the intersection of the $J^{\prime}$-stuttering facets of the simplex corresponding to this particular representative will be non-empty and lie in the desired intersection $F$. Given this claim, we can describe $\Delta_{\omega, J}$ as the sub-complex of the boundary of the polytope $\Delta_{\omega}$ which is the union of all facets not containing the face $F$. This implies $\Delta_{\omega, J}$ is a ball by Lemma 6.4. This completes the proof of Lemma 3.5.

To complete the proof of assertion (2) in Lemma 3.3, we need to show that $\Delta_{m, J}$ is homotopy equivalent to the one-point wedge of spheres $\bigvee_{\omega \in \mathcal{C}(m)} \mathbf{S}^{e-3}$. To see this, we apply the Wedge Lemma 6.1 to the covering $\mathcal{U}_{m, J}$. This covering satisfies the necessary hypotheses because all of the spaces $U_{p}$ for $p$ non-minimal in $P$ coincide with the corresponding spaces in the covering 
$\mathcal{U}_{m}$ (which came from a polytopal decomposition of a polytopal ball). Let $P$ denote the common intersection poset of $\mathcal{U}_{m}$ and $\mathcal{U}_{m, J}$. Note that in $\mathcal{U}$, for any $p$ in $P$ with $p \neq \hat{1}$, the corresponding space (which is the same as in $\left.\mathcal{U}_{m, J}\right)$ is contractible because it is a polytope. Also note that the order complex $\Delta\left(P_{<\hat{1}}\right)$ is contractible because $P_{<\hat{1}}$ is the face poset for the polytopal decomposition of the ball $\Delta_{m}$, and hence it triangulates the barycentric subdivision of this polytopal decomposition.

Therefore $\Delta_{m, J}$ is homotopy equivalent to the wedge

$$
\bigvee_{p \in P} \Delta\left(P_{<p}\right) * U_{p}
$$

By the previous discussion, the only non-contractible contributions to the wedge come from the spaces $U_{p}$ corresponding to the minimal elements $p$ in the covering. But these spaces $U_{p}$ are by definition the spaces $\Delta_{\omega, J}$. Lemma 3.5 then gives exactly what we want, and the proof of Lemma 3.3 is complete.

\section{Topological proofs of Koszulness.}

In this section we specifically study the following two situations and give topological proofs of Koszulness of $R$ :

- $\Lambda=\mathbf{N}^{d}$ (so $R=k\left[x_{1}, \ldots, x_{d}\right]$ ) and $J$ is a quadratic monomial ideal, or

- $J=0$ and $\Lambda$ has minimal generators $\alpha_{1}, \ldots, \alpha_{n}$ for which the toric ideal $I_{\Lambda}$ has a quadratic Gröbner basis. We refer the reader to [CLO] for notions and terminology related to Gröbner bases.

Let $S$ be an $\mathbf{N}^{d}$-graded finitely generated associative $k$-algebra. The Hilbert series of $S$ is the generating function

$$
\operatorname{Hilb}\left(R ; z_{1}, \ldots, z_{d}\right)=\sum_{\lambda \in \mathbf{N}^{d}} \operatorname{dim}_{k}\left(S_{\lambda}\right) \mathbf{z}^{\lambda},
$$

although we will occasionally use other variables than $z_{1}, \ldots, z_{n}$ as power series variable in the generating function. In fact, we will even abuse notation by sometimes using the variables $x_{i}$ or $z_{i}$ as generating function variables when $S$ is a sub-algebra or quotient of $k\left[x_{1}, \ldots, x_{n}\right]$ or $k\left[z_{1}, \ldots, z_{d}\right]$. We hope that this creates no confusion.

As was mentioned earlier, the groups $\operatorname{Tor}_{i}^{R}(k, k)$ are $\mathbf{N}^{d}$-graded or $\Lambda$ graded as well. Let $\operatorname{Tor}_{i}^{R}(k, k)_{\lambda}$ be the $\lambda$-graded part of $\operatorname{Tor}_{i}^{R}(k, k)$. The Poincaré series of $k$ is

$$
\operatorname{Poin}_{R}^{k}\left(t, z_{1}, \ldots, z_{d}\right)=\sum_{i \geq 0} \sum_{\lambda \in \mathbf{N}^{d}} \operatorname{dim}_{k} \operatorname{Tor}_{i}^{R}(k, k)_{\lambda} t^{i} \mathbf{z}^{\lambda} .
$$


Let $R=k[\Lambda] / J$ be an affine semigroup ring for a semigroup $\Lambda \subseteq \mathbf{N}^{d}$ modulo a semigroup ideal $J$. Then clearly

$$
\operatorname{Hilb}(R ; \mathbf{z}):=\sum_{\lambda \in \Lambda} \operatorname{dim}_{k} R_{\lambda} \mathbf{z}^{\lambda}=\sum_{\lambda \in \Lambda \backslash J} \mathbf{z}^{\lambda} .
$$

We first recover Fröberg's result:

Corollary $4.1([\mathrm{Fr} 1])$. Let $R=k\left[x_{1}, \ldots, x_{n}\right] / J$ for a quadratic monomial ideal $J$. Then $R$ is Koszul for all fields $k$, and

$$
\begin{aligned}
\operatorname{Poin}_{R}^{k}(t, \mathbf{x}) & =\operatorname{Hilb}\left(k\left\langle y_{1}, \ldots, y_{n}\right\rangle /\left(\left\{y_{i} y_{j}-y_{j} y_{i}\right\}_{x_{i} x_{j} \notin J},\left\{y_{i}^{2}\right\}_{x_{i}^{2} \notin J}\right) ; t, \mathbf{x}\right) \\
& =\left[\frac{1}{\operatorname{Hilb}(R ; \mathbf{x})}\right]_{x_{i} \mapsto-t x_{i}}
\end{aligned}
$$

where the non-commutative variable $y_{i}$ is considered to have the same multidegree as $x_{i}$, and here $x_{i}$ is used both as a variable in the polynomial ring and as an indeterminate in the Hilbert series.

Proof. Equation (4.2) follows from Koszulness of $R$ (see Lemma 5.5). Koszulness of $R$ and Equation (4.1) will follow from Theorem 2.1, once we establish that for any monomial $m$ in $R$ of degree $e$ we have

$$
\tilde{\mathrm{H}}_{i-2}\left(\Delta_{m}, \Delta_{m, J} ; k\right)= \begin{cases}k^{|\mathcal{C}(m)|} & \text { if } i=e \\ 0 & \text { else. }\end{cases}
$$

To see this in the case where $m$ is square-free of degree $e$, by Theorem 3.1 we need to show that

$$
\tilde{\mathrm{H}}_{i-2}\left(\mathbf{S}^{e-2}, \mathbf{S}^{e-2} \cap \mathcal{A}_{J^{e}} ; k\right)= \begin{cases}k^{|\mathcal{C}(m)|} & \text { if } i=e \\ 0 & \text { else. }\end{cases}
$$

However, this follows from Lemma 3.2, as we now explain. Note that the relative homology of $\left(\mathbf{S}^{e-2}, \mathbf{S}^{e-2} \cap \mathcal{A}_{J^{e}}\right)$ is the same as the (absolute) homology of the quotient space $\mathbf{S}^{e-2} /\left(\mathbf{S}^{e-2} \cap \mathcal{A}\right)$, because the two spaces form a relative pair of finite $\mathrm{CW}$-complexes. On the other hand, for any hyperplane arrangement $\mathcal{A}$ in an $(e-1)$-dimensional space, the quotient space $\mathbf{S}^{e-2} /\left(\mathbf{S}^{e-2} \cap \mathcal{A}\right)$ will be the one-point wedge of spheres $\bigvee_{\omega \in \mathcal{C}} \mathbf{S}^{e-2}$ indexed by the set of chambers $\mathcal{C}$ cut out by $\mathcal{A}$. Therefore Lemma 3.2 gives the assertion. 
In the case $m$ is not square-free, $\Delta_{m}$ is a ball by Lemma 3.3. Thus the long exact sequence for homology gives

$$
\begin{aligned}
\tilde{\mathrm{H}}_{i-2}\left(\Delta_{m}, \Delta_{m, I} ; k\right) & \cong \tilde{\mathrm{H}}_{i-3}\left(\Delta_{m, I} ; k\right) \\
& \cong \tilde{\mathrm{H}}_{i-3}\left(\bigvee_{\omega \in \mathcal{C}(m)} \mathbf{S}^{e-3} ; k\right) \\
& = \begin{cases}k^{|\mathcal{C}(m)|} & \text { if } i=e \\
0 & \text { else, }\end{cases}
\end{aligned}
$$

where the second isomorphism also follows from Lemma 3.3.

For the rest of this section, we consider the case when the toric ideal $I_{\Lambda}$ has a quadratic initial ideal with respect to some monomial ordering $\prec$ on $k\left[x_{1}, \ldots, x_{n}\right]$. Let $J$ be the initial ideal $J=i n_{\prec} I_{\Lambda}$.

Extending the notation of $\S 3$, given $\lambda$ in $\Lambda$, say that a monomial $m$ in $k\left[x_{1}, \ldots, x_{n}\right]$ is in the commutative fiber of $\lambda$ if it maps to $\mathbf{z}^{\lambda}$ under $x_{i} \mapsto \mathbf{z}^{\alpha_{i}}$, and say that a monomial in $k\left\langle y_{1}, \ldots, y_{n}\right\rangle$ is in the non-commutative fiber of $\lambda$ if it maps to an element of the commutative fiber of $\lambda$ under $y_{i} \mapsto x_{i}$. Note that the facets (maximal faces) of $\Delta_{\lambda}$ correspond to maximal chains in the open interval poset $(0, \lambda)$ and therefore naturally biject with the monomials $\lambda$ in the non-commutative fiber of $\lambda$.

Say that the monomial ordering $\prec$ reversely grades $\Lambda$ if whenever $m, m^{\prime}$ lie in the commutative fiber of some $\lambda \in \Lambda$ and $m \prec m^{\prime}$ we have $\operatorname{deg}(m) \geq$ $\operatorname{deg}\left(m^{\prime}\right)$.

As in Section 3, for any ideal $J$ generated by quadratic monomials, we have a notion of $J^{\prime}$-commuting equivalence classes and $J^{\prime}$-non-stuttering for non-commutative monomials. Denote by $\mathcal{C}(\lambda)$ the set of $J^{\prime}$-non-stuttering $J^{\prime}$-commuting equivalence classes in the non-commutative fiber of $\lambda$.

Theorem 4.2. Let $\Lambda \subseteq \mathbf{N}^{d}$ be an affine semigroup. Assume that either

- $I_{\Lambda}$ is homogeneous with respect to $\operatorname{deg}\left(x_{i}\right)=1$ and there is a term order for which it has a Gröbner basis of quadratic binomials, or

- $I_{\Lambda}$ is not homogeneous with respect to the above grading and there is a term order $\prec$ which reversely grades $\Lambda$ and for which $I_{\Lambda}$ has a quadratic initial ideal.

Then the simplicial complex $\Delta_{\lambda}$ is homotopy equivalent to the wedge of spheres

$$
\bigvee_{\omega \in \mathcal{C}(\lambda)} \mathbf{S}^{|\omega|-2}
$$


where $|\omega|$ is the length of any of the non-commutative monomials in its equivalence class.

Proof. For each monomial $m$ in the commutative fiber of $\lambda$, let $\Delta_{m}$ be the sub-complex of $\Delta_{\lambda}$ generated by the maximal faces corresponding to noncommutative monomials $\lambda$ of content $m$. It follows from Theorem 3.1 and Lemma 3.3 that $\Delta_{m}$ is either a sphere $\mathbf{S}^{\operatorname{deg}(m)-2}$ or a ball $\mathbf{B}^{\operatorname{deg}(m)-2}$ depending upon whether or not $m$ is square-free.

Order the monomials $m_{1}, m_{2}, \ldots, m_{r}$ in the commutative fiber of $\lambda$ according to $\prec$, i.e.

$$
m_{1} \prec \cdots \prec m_{r} .
$$

Let $J$ be the initial ideal of $I_{\Lambda}$ with respect to $\prec$. For a fixed $s \leq r$, if we let $X=\bigcup_{i=1}^{s-1} \Delta_{m_{i}}$ and $Y=\Delta_{m_{s}}$, it is then easy to check that $X \cap Y$ may be identified with the sub-complex $\Delta_{m_{s}, J}$ in the notation of Theorem 3.1. By induction on $s$, we have that $X$ is homotopy equivalent to $\bigvee_{\omega} \mathbf{S}^{|\omega|-2}$ as $\omega$ ranges over the $J^{\prime}$-non-stuttering $J^{\prime}$-commuting classes of monomials having content in $\left\{m_{1}, \ldots, m_{s-1}\right\}$. Now we consider the change in topology from $X$ to $X \cup Y$ in the inductive step when we add in $Y=\Delta_{m_{s}}$. We will see below that the intersection $X \cap Y$ is always homotopy equivalent to a wedge of spheres of codimension 1 in $Y$, and then the assumption that $\prec$ reversely grades $\Lambda$ insures that the hypotheses in Lemma 6.2 about dimensions are satisfied. In order to analyze which spheres occur in the wedge, we break up into two cases depending on whether $m_{s}$ is square-free.

If $m_{s}$ is square-free of degree $e$, then $Y=\mathbf{S}^{e-2}$ and $X \cap Y \cong \mathbf{S}^{e-2} \cap \mathcal{A}_{J^{e}}$. By Lemma 6.3 and Lemma 3.2, we have

$$
X \cap Y=\bigvee_{j=1}^{\left|\mathcal{C}\left(m_{s}\right)\right|-1} \mathbf{S}^{e-3} .
$$

Hence by induction on $s$ and Lemma 6.2 we have

$$
X \cup Y=\bigvee_{\omega} \mathbf{S}^{|\omega|-2}
$$

as $\omega$ ranges over the $J^{\prime}$-non-stuttering $J^{\prime}$-commuting classes of monomials having content in $\left\{m_{1}, \ldots, m_{s}\right\}$. This completes the inductive step in this case.

If $m_{s}$ is not square-free, then $Y \cong \mathbf{B}^{\operatorname{deg}\left(m_{s}\right)-2}$ and Lemma 3.3 says that $X \cap Y$ is homotopy equivalent to $\bigvee_{j=1}^{\left|\mathcal{C}\left(m_{s}\right)\right|} \mathbf{S}^{\operatorname{deg}\left(m_{s}\right)-2}$. Hence by induction and Lemma 6.2 we have

$$
X \cup Y=\bigvee_{\omega} \mathbf{S}^{|\omega|-2}
$$


as $\omega$ ranges over the $J^{\prime}$-non-stuttering $J^{\prime}$-commuting classes of monomials with content in $\left\{m_{1}, \ldots, m_{s}\right\}$. This completes the inductive step in the remaining case, so the theorem is proved.

Corollary 4.3. With $\Lambda, R, I_{\Lambda}, J$ as in the previous result, we have

$$
\begin{aligned}
\operatorname{Poin}_{R}^{k}(t, \mathbf{z}) & =\operatorname{Hilb}\left(k\left\langle y_{1}, \ldots, y_{n}\right\rangle /\left(\left\{y_{i} y_{j}-y_{j} y_{i}\right\}_{x_{i} x_{j} \notin J},\left\{y_{i}^{2}\right\}_{x_{i}^{2} \notin J}\right) ; t, \mathbf{z}\right) \\
& =\left[\frac{1}{\operatorname{Hilb}\left(k\left[x_{1}, \ldots, x_{n}\right] / J ; \mathbf{x}\right)}\right]_{x_{i} \mapsto-t \mathbf{z}^{\alpha_{i}}} \cdot
\end{aligned}
$$

Furthermore:

- If $I_{\Lambda}$ is homogeneous with respect to $\operatorname{deg}\left(x_{i}\right)=1$, then $R$ is Koszul and all of the complexes $\Delta_{\lambda}$ are Cohen-Macaulay for any field $k$, i.e. $\Lambda$ is a Cohen-Macaulay poset.

- If $I_{\Lambda}$ is not homogeneous and there is a term order $\prec$ which reversely grades $\Lambda$ and for which $I_{\Lambda}$ has a quadratic initial ideal, then all of the complexes $\Delta_{\lambda}$ are sequentially Cohen-Macaulay for any field $k$ (see [Wa] for a definition and facts about sequential Cohen-Macaulayness).

Proof. By Theorems 2.1 and 4.2 we have that

$$
\begin{aligned}
\operatorname{Poin}_{R}^{k}(t, \mathbf{z}) & =\sum_{\lambda \in \Lambda, i \geq 0} \operatorname{dim}_{k} \operatorname{Tor}_{i}^{R}(k, k)_{\lambda} t^{i} \mathbf{z}^{\lambda}=\sum_{\lambda \in \Lambda, i \geq 0} \operatorname{dim}_{k} \tilde{\mathrm{H}}_{i-2}\left(\Delta_{\lambda} ; k\right) t^{i} \mathbf{z}^{\lambda} \\
& =\sum_{\lambda \in \Lambda, i \geq 0} \operatorname{dim}_{k} \tilde{\mathrm{H}}_{i-2}\left(\bigvee_{\omega \in \mathcal{C}(\lambda)} \mathbf{S}^{|\lambda|-2} ; k\right) t^{i} \mathbf{z}^{\lambda} \\
& =\sum_{\lambda \in \Lambda} \sum_{\omega \in \mathcal{C}(\lambda)} t^{|\omega|} z^{\lambda} \\
& =\operatorname{Hilb}\left(k\left\langle y_{1}, \ldots, y_{n}\right\rangle /\left(\left\{y_{i} y_{j}-y_{j} y_{i}\right\}_{x_{i} x_{j} \notin J},\left\{y_{i}^{2}\right\}_{x_{i}^{2} \notin J}\right) ; t, \mathbf{z}\right) .
\end{aligned}
$$

This proves the first equality in the corollary. Equality (4.3) then follows from Equation (4.2).

In the homogeneous case, Koszulness follows from Theorem 4.2 since every $\lambda$ in $\mathcal{C}(\lambda)$ has the same length $|\lambda|$. Cohen-Macaulayness of $\Delta_{\lambda}$ follows from Theorem 4.2 and the fact that all links of its faces are joins of complexes of the form $\Delta_{\mu}$ for $\mu$ in $\Lambda$.

In the inhomogeneous case, sequential Cohen-Macaulayness again follows from Theorem 4.2 and [Wa, Theorem 1.3] along with the fact that all links of faces in $\Delta_{\lambda}$ are joins of complexes of the same form.

One of the motivating special cases for these results are the 2-dimensional normal affine semigroup rings whose Poincaré series were studied in [LS] 
and further studied in [PRS]. In this case $\Lambda$ is the set of points in $\mathbf{N}^{2}$ which lie in a closed 2-dimensional cone bounded by two rays of rational slope emanating from the origin (see proof of Proposition 5.3). If one numbers the minimal generators $\alpha_{1}, \ldots, \alpha_{n}$ of $\Lambda$ as they are traversed in counterclockwise order from the $x$-axis to the $y$-axis, then it is easy to check that the reverse lexicographic order $\prec$ reversely grades $\Lambda$ and gives a quadratic initial ideal $J$.

We remark that in the next section, it will be shown that in the inhomogeneous case of Corollary 4.3, $R$ is Koszul in a more generalized sense to be defined.

\section{Koszulness.}

In this section we extend the notion of a Koszul algebra to local and $\mathbf{N}^{d}$ graded $k$-algebras (Definition 5.1). Our motivation for this definition was to understand algebraically Equation (4.3) in the previous section, and this is achieved in Proposition 5.4 (see also [Fr2] for a formulation in the local situation) and the results following it. Proposition 5.4 shows that for rings $R$ which are Koszul in our more general sense, one can again relate the Poincaré series to the Hilbert series of its associated graded ring, and thereby obtain a rational expression for the Poincare series. One application is that this more general notion of Koszulness is shown to apply to all Cohen-Macaulay rings of minimal multiplicity (Theorem 5.2).

Throughout this section $(R, \mathfrak{m})$ denotes either

- a Noetherian local ring $R$ with maximal ideal $\mathfrak{m}$, or

- an $\mathbf{N}^{d}$-graded $k$-algebra $R$ with maximal graded ideal $\mathfrak{m}:=\bigoplus_{\substack{\lambda \in \mathbb{N}^{d} \\ \lambda \neq 0}} R_{\lambda}$.

We will generally state results for both kinds of rings, but often give proofs only in the case of local rings when the proofs for $\mathbf{N}^{d}$-graded rings are analogous.

Let $M$ be an $R$-module (respectively, graded $R$-module). Then we denote by $\operatorname{gr}_{\mathfrak{m}}(M)$ the associated graded module $\bigoplus_{i \geq 0} \mathfrak{m}^{i} M / \mathfrak{m}^{i+1} M$ with respect to $\mathfrak{m}$. In particular, the associated graded ring $\operatorname{gr}_{\mathfrak{m}}(R)$ is an $\mathbf{N}$-graded $k$-algebra.

Definition 5.1. We call a ring $(R, \mathfrak{m})$ as above $K o s z u l$, if $\operatorname{gr}_{\mathfrak{m}}(R)$ is Koszul in the usual sense for $\mathbf{N}$-graded rings, i.e. if $\operatorname{Tor}_{i}^{\mathrm{gr}_{\mathrm{m}}}(R)(k, k)_{j}=0$ for $j \neq i$.

Note that in the case where $R$ is an $\mathbf{N}$-graded $k$-algebra, then $\operatorname{gr}_{\mathfrak{m}}(R) \cong R$ as $\mathbf{N}$-graded rings. Thus our definition is an extension of the usual notion of Koszulness (see also [Fr2]).

Before exploring the consequences of Koszulness for $R$, we exhibit a class of rings which are Koszul in this generalized sense, but we must first recall some basic notions of commutative algebra. 
A regular sequence for $(R, \mathfrak{m})$ is a sequence $\left(\theta_{1}, \ldots, \theta_{r}\right)$ of elements in $\mathfrak{m}$ such that $\theta_{i+1}$ is a non-zerodivisor in $R /\left(\theta_{1}, \ldots, \theta_{i}\right)$. A local ring $(R, \mathfrak{m})$ is called Cohen-Macaulay if there exists a regular sequence $\left(\theta_{1}, \ldots, \theta_{r}\right)$ in $R$ of length $r$ equal to the Krull dimension $\operatorname{dim} R$.

When $R$ is a Noetherian $\mathbf{N}$-graded $k$-algebra, its Hilbert series

$$
\operatorname{Hilb}(R ; t):=\sum_{i \geq 0} \operatorname{dim}_{k} R_{i} t^{i}
$$

is known to be a rational function $P(t) / Q(t)$ by a fundamental result of Hilbert and Serre. The multiplicity of $R$ is defined to be $e(R):=P(1)$ when $P, Q$ are chosen so that $\operatorname{gcd}(P(t), Q(t))=1$. For our local and $\mathbf{N}^{d}$ graded rings $(R, \mathfrak{m})$, we define more generally the multiplicity $e(R)$ of $R$ as $e\left(\operatorname{gr}_{\mathfrak{m}}(R)\right)$; see [BH1, p. 181].

Recall (see $[\mathbf{A b}])$ that if $(R, \mathfrak{m})$ is Cohen-Macaulay, then the multiplicity $e(R)$ is bounded from below as follows:

$$
e(R) \geq \operatorname{dim}_{k} \mathfrak{m} / \mathfrak{m}^{2}-\operatorname{dim}(R)+1
$$

where $\operatorname{dim}_{k} \mathfrak{m} / \mathfrak{m}^{2}$ is called the embedding dimension of $(R, \mathfrak{m})$. In the case of equality one says that the ring has minimal multiplicity (see [BH1, Exercise 4.5.14]).

Theorem 5.2. If $\Lambda$ is an affine semigroup in $\mathbf{N}^{d}$ and $k[\Lambda]$ is CohenMacaulay with minimal multiplicity then $k[\Lambda]$ is Koszul. In particular, if $k[\Lambda]$ is normal with minimal multiplicity then $k[\Lambda]$ is Koszul in the generalized sense.

Proof. The second assertion follows from the first, since by a result of Hochster every normal affine semigroup ring is Cohen-Macaulay; see [BH1, Theorem 6.3.5 (a)].

By a result of J.D. Sally [Sa], if $R$ is Cohen-Macaulay and of minimal multiplicity then $\operatorname{gr}_{\mathfrak{m}}(R)$ is Cohen-Macaulay too. It also follows that $\operatorname{gr}_{\mathfrak{m}}(R)$ is of minimal multiplicity, since $\operatorname{gr}_{\mathfrak{m}}(R)$ and $R$ share the same Krull dimension [BH1, Theorem 4.4.6 (b)], and clearly also the same embedding dimension. Therefore, we may replace $R$ by $\operatorname{gr}_{\mathfrak{m}}(R)$ and assume that we have the following situation: $R$ is $\mathbf{N}$-graded, generated by $R_{1}$, and Cohen-Macaulay of minimal multiplicity. We may also assume that $k$ is an infinite field, since one can easily check that the Koszul property is independent of flat ring extensions. Hence, there exists a maximal regular sequence $\theta_{1}, \ldots, \theta_{r}$ of 1-forms in $R$ such that $\mathfrak{m}^{2}=\left(\theta_{1}, \ldots, \theta_{r}\right) \mathfrak{m}$; see [BH1, Exercise 4.5.14 (c)]. Thus, the square of the maximal ideal of $A=R /\left(\theta_{1}, \ldots, \theta_{r}\right)$ is zero, which implies that

$$
A \cong k\left[x_{1}, \ldots, x_{m}\right] /\left(x_{i} x_{j}\right)_{1 \leq i \leq j \leq m}
$$


Therefore, by the result of Fröberg (Corollary 4.1) the ring $A$ is Koszul. However, it is known that the quotient of a homogeneous $k$-algebra by a regular sequence of 1 -forms is Koszul if and only if the algebra itself is Koszul. This can be easily seen from the fact that if $Q$ is an $\mathbf{N}$-graded $k$-algebra and $\theta$ is a non-zero divisor of degree 1 then

$$
\operatorname{Poin}_{Q}^{k}(t, z):=\sum_{i, j \geq 0} \operatorname{dim}_{k} \operatorname{Tor}_{i}^{Q}(k, k)_{j} t^{i} z^{j}=\operatorname{Poin}_{Q /(\theta)}^{k}(t, z) \cdot(1+t z) .
$$

Hence $Q$ is Koszul if and only if $Q /(\theta)$ is. Applying this fact repeatedly to $R$ and the regular sequence $\theta_{1}, \ldots, \theta_{r}$, we conclude that $R$ is Koszul.

Since much of this work was originally motivated by the study of 2dimensional normal affine semigroup rings, it is worth mentioning the following fact:

Proposition 5.3. All 2-dimensional normal affine semigroup rings are Cohen-Macaulay of minimal multiplicity, and hence Koszul in the generalized sense.

Proof. Assume $\Lambda \subseteq \mathbf{N}^{2}$ is normal. We know that $R=k[\Lambda]$ is CohenMacaulay because it is normal. By Gordan's Lemma (see Proposition 6.1.2 [BH1]) we can assume that $\Lambda$ is the set of integral points in the intersection of two rational halfspaces. Recall, that in the given situation the multiplicity $e(R)$ is counted by the normalized volume (=twice the usual volume) of the set-theoretic difference of the convex hull of $\Lambda$ and the convex hull of $\Lambda \backslash\{\mathbf{0}\}$ (see Chapter 5.3 of [GKZ]). This set is triangulated by triangles whose only integral points are the vertices, which consist of two neighboring minimal generators of $\Lambda$ and the origin. Each of these triangles has volume $1 / 2$. The number of triangles equals the number of minimal generators of $\Lambda$ minus 1 . But this implies that $k[\Lambda]$ is of minimal multiplicity.

We now proceed to investigate the consequences of Koszulness for $(R, \mathfrak{m})$. Proposition 5.4 tells us that $R$ and $\operatorname{gr}_{\mathfrak{m}}(R)$ share the same $\left(\mathbf{N}^{d}\right.$-graded) Poincaré series, when $(R, \mathfrak{m})$ is Koszul. In particular, this means that $\operatorname{gr}_{\mathfrak{m}}(R)$ is Koszul in the usual sense, and hence both its own Poincaré series and that of $R$ are rational. Before we can discuss this, however, we must first make clear what is meant by the various gradings, Poincaré series, and Hilbert series for $\operatorname{gr}_{\mathfrak{m}}(R)$ when $R$ is $\mathbf{N}^{d}$-graded.

Given $(R, \mathfrak{m})$ with a $\mathbf{N}^{d}$-grading on $R$, we know that $\operatorname{gr}_{\mathfrak{m}}(R)$ carries a natural $\mathbf{N}$-grading in which the degree of a homogeneous element $f$ is the number $i$ such that $f \in \mathfrak{m}^{i} \backslash \mathfrak{m}^{i+1}$. We also define a $\mathbf{N}^{d}$-grading on 
$\operatorname{gr}_{\mathfrak{m}}(R)$ as follows. An element $g+\mathfrak{m}^{i+1}, g \in \mathfrak{m}^{i} \backslash \mathfrak{m}^{i+1}$ is called $\mathbf{N}^{d}$ homogeneous of degree $a=\left(a_{1}, \ldots, a_{d}\right)$, if there exists an $\mathbf{N}^{d}$ homogeneous element $h \in R$ of degree $a$ such that $g+\mathfrak{m}^{i}=h+\mathfrak{m}^{i}$. One easily checks that this is well-defined and induces an $\mathbf{N}^{d}$-grading on $\operatorname{gr}_{\mathfrak{m}}(R)$ that is compatible with the multiplication and that $\operatorname{gr}_{\mathfrak{m}}(R)$ is the direct sum of its multigraded components. Putting together the two gradings ( $\mathbf{N}-$ and $\mathbf{N}^{d}-$ ) on $\operatorname{gr}_{\mathfrak{m}}(R)$ gives a $\mathbf{N}^{d+1}$-grading, and we can consider the finely graded Hilbert series $\operatorname{Hilb}\left(\operatorname{gr}_{\mathfrak{m}}(R), z_{0}, z_{1}, \ldots, z_{d}\right)$ in which the first variable $z_{0}$ keeps track of the $\mathbf{N}$-degree. The Poincaré series $\operatorname{Poin}_{R}^{k}\left(t, z_{1}, \ldots, z_{d}\right)$ and $\operatorname{Poin}_{\mathrm{gr}_{\mathrm{m}}(R)}^{k}\left(t, z_{0}, z_{1}, \ldots, z_{d}\right)$ are then defined as usual (i.e. with $t$ keeping track of the homological degree). In the local case, there is no ambiguity in defining $\operatorname{Poin}_{R}^{k}(t)$, and we define $\operatorname{Hilb}\left(\operatorname{gr}_{\mathfrak{m}}(R) ; z\right), \operatorname{Poin}_{\mathrm{gr}_{\mathfrak{m}}(R)}(t, z)$ similarly to the above.

The following result is due to Fröberg [Fr2], where it is stated in the local situation. We give a different proof which also exhibits more facts relating the resolution of $k$ over $R$ and over $\operatorname{gr}_{\mathfrak{m}}(R)$ (see Lemma 5.8).

Proposition 5.4. If $(R, \mathfrak{m})$ is Koszul and $\mathbf{N}^{d}$-graded we have for all $\lambda \in \mathbf{N}^{d}$

$$
\operatorname{Tor}_{i}^{R}(k, k)_{\lambda} \cong \operatorname{Tor}_{i}^{\mathrm{gr}}(R)(k, k)_{\lambda}
$$

and consequently

$$
\operatorname{Poin}_{R}^{k}\left(t, z_{1}, \ldots, z_{d}\right)=\left[\operatorname{Poin}_{\mathrm{gr}_{\mathfrak{m}}(R)}^{k}\left(t, z_{0}, z_{1}, \ldots, z_{d}\right)\right]_{z_{0} \mapsto 1} .
$$

If $(R, \mathfrak{m})$ is Koszul and local we have

$$
\operatorname{Tor}_{i}^{R}(k, k) \cong \operatorname{Tor}_{i}^{\mathrm{gr}}(R)(k, k)
$$

and consequently

$$
\operatorname{Poin}_{R}^{k}(t)=\left[\operatorname{Poin}_{\mathrm{gr}_{\mathfrak{m}}(R)}^{k}(t, z)\right]_{z \mapsto 1} .
$$

The isomorphism of the Tor-groups asserted in the theorem will be proved in two lemmas (Lemma 5.7 and Lemma 5.8) at the end of this section. But first let us deduce some consequences of Proposition 5.4. The first consequence requires a simple lemma:

Lemma 5.5. Let $Q$ be a $\mathbf{N}^{d+1}$-graded $k$-algebra which is Koszul (in the standard sense) with respect to the $\mathbf{N}$-grading induced by the first component of its $\mathbf{N}^{d+1}$-grading. Set

$$
\operatorname{Hilb}\left(Q ; z_{0}, z_{1}, \ldots, z_{d}\right):=\sum_{\left(a_{0}, a_{1}, \ldots, a_{d}\right) \in \mathbf{N}^{d+1}} \operatorname{dim}_{k} R_{\left(a_{0}, a_{1}, \ldots, a_{d}\right)} z_{0}^{a_{0}} z_{1}^{a_{1}} \cdots z_{d}^{a_{d}}
$$


and

$$
\begin{aligned}
\operatorname{Poin}_{Q}^{k}\left(t, z_{1}, \ldots, z_{d}\right) \\
=\sum_{i \geq 0} t^{i} \sum_{\left(a_{0}, a_{1}, \ldots, a_{d}\right) \in \mathbf{N}^{d+1}} \operatorname{dim}_{k} \operatorname{Tor}_{i}^{R}(k, k)_{\left(a_{0}, a_{1}, \ldots, a_{d}\right)} z_{1}^{a_{1}} \cdots z_{d}^{a_{d}} .
\end{aligned}
$$

Then

$$
\operatorname{Poin}_{Q}^{k}\left(t, z_{1}, \ldots, z_{d}\right)=\left[\frac{1}{\operatorname{Hilb}\left(Q ; z_{0}, z_{1}, \ldots, z_{d}\right)}\right]_{z_{0} \mapsto-t} .
$$

Proof. It is easy to see that one can choose a minimal free resolution of $Q$ in which the basis elements and maps are all $\mathbf{N}^{d+1}$-graded. Interpreting the dimensions of the multigraded components of $\operatorname{Tor}^{Q}(k, k)$ as the multidegrees of the basis elements in the free modules of the resolution, exactness of the resolution gives

$$
\begin{aligned}
1=\sum_{i \geq 0}(-1)^{i} \sum_{\left(a_{0}, \ldots, a_{d}\right) \in \mathbf{N}^{d+1}} \operatorname{dim}_{k} \operatorname{Tor}_{i}^{Q}(k, k)_{\left(a_{0}, \ldots, a_{d}\right)} z_{0}^{a_{0}} z_{1}^{a_{1}} \cdots z_{d}^{a_{d}} \\
\cdot \operatorname{Hilb}\left(Q ; z_{0}, z_{1}, \ldots, z_{d}\right) .
\end{aligned}
$$

Since $Q$ is Koszul with respect to the first grading variable we conclude that only the terms with $i=a_{0}$ in the above sum are non-zero. Hence

$$
\begin{aligned}
1=\sum_{i \geq 0}\left(-z_{0}\right)^{i} \sum_{\left(a_{0}, \ldots, a_{d}\right) \in \mathbf{N}^{d+1}} \operatorname{dim}_{k} \operatorname{Tor}_{i}^{Q}(k, k)_{\left(a_{0}, \ldots, a_{d}\right)} z_{1}^{a_{1}} \cdots z_{d}^{a_{d}} \\
\cdot \operatorname{Hilb}\left(Q ; z_{0}, z_{1}, \ldots, z_{d}\right) .
\end{aligned}
$$

The last equation is equivalent to the assertion of the lemma.

Corollary 5.6. Let $(R, \mathfrak{m})$ be Koszul in the generalized sense. Then if $R$ is $\mathbf{N}^{d}$-graded we have

$$
\operatorname{Poin}_{R}^{k}\left(t, z_{1}, \ldots, z_{d}\right)=\left[\frac{1}{\operatorname{Hilb}\left(\operatorname{gr}_{\mathfrak{m}}(R) ; z_{0}, z_{1}, \ldots, z_{d}\right)}\right]_{z_{0} \mapsto-t},
$$

and if $R$ is local we have

$$
\operatorname{Poin}_{R}^{k}(t)=\frac{1}{\operatorname{Hilb}\left(\operatorname{gr}_{\mathfrak{m}}(R) ;-t\right)}
$$

In particular, in either case the Poincaré series for $R$ is rational.

Proof. Apply Proposition 5.4, and then Lemma 5.5 with $Q=\operatorname{gr}_{\mathfrak{m}}(R)$.

We are now in a position to deduce Equation (4.3) algebraically. 
Algebraic proof of Equation (4.3). We use the same notation as in Corollary 4.3. Recall that the toric ideal $I_{\Lambda}$ is the kernel of the surjection $k[\mathbf{x}] \rightarrow R=k[\Lambda]$ defined by $x_{i} \mapsto \mathbf{z}^{\alpha_{i}}$. Similarly, define $L_{\Lambda}$ to be the kernel of the surjection $k[\mathbf{x}] \rightarrow \operatorname{gr}_{\mathfrak{m}}(R)$ defined by $x_{i} \mapsto \overline{\mathbf{z}^{\alpha_{i}}}$. It then follows by definition of $\operatorname{gr}_{\mathfrak{m}}(R)$ that

$$
L_{\Lambda}=i n_{\mathfrak{m}}\left(I_{\Lambda}\right):=\left(i n_{\mathfrak{m}}(f): f \in I_{\Lambda}\right)
$$

where here $i n_{\mathfrak{m}}(f)$ is the term in $f$ having smallest degree in the $\mathbf{N}$-grading induced by powers of $\mathfrak{m}$. Also, since $\prec$ reversely grades $\Lambda$,

$$
i n_{\prec}\left(L_{\Lambda}\right)=i n_{\prec}\left(i n_{\mathfrak{m}}\left(I_{\Lambda}\right)\right)=i n_{\prec}\left(I_{\Lambda}\right)=: J .
$$

As a consequence, since $J$ is assumed to be a quadratic monomial ideal, we conclude that $L_{\Lambda}$ has the same quadratic initial ideal $J$ with respect to $\prec$ that $I_{\Lambda}$ did. Hence $\operatorname{gr}_{\mathfrak{m}}(R)$ is Koszul and $R$ is Koszul in the generalized sense. We then have the following chain of equalities

$$
\begin{aligned}
& \operatorname{Poin}_{R}^{k}(t, \mathbf{z}) \\
& =\left[\frac{1}{\operatorname{Hilb}\left(\operatorname{gr}_{\mathfrak{m}}(R) ; z_{0}, z_{1}, \ldots, z_{d}\right)}\right]_{z_{0} \mapsto-t}=\left[\frac{1}{\operatorname{Hilb}\left(k[\mathbf{x}] / L_{\Lambda} ; \mathbf{x}\right)}\right]_{x_{i} \mapsto-t \mathbf{z}^{\alpha_{i}}} \\
& =\left[\frac{1}{\operatorname{Hilb}\left(k[\mathbf{x}] / i n_{\prec}\left(L_{\Lambda}\right) ; \mathbf{x}\right)}\right]_{x_{i} \mapsto-t \mathbf{z}^{\alpha_{i}}}=\left[\frac{1}{\operatorname{Hilb}(k[\mathbf{x}] / J ; \mathbf{x})}\right]_{x_{i} \mapsto-t \mathbf{z}^{\alpha_{i}}} .
\end{aligned}
$$

The first equality is by Corollary 5.6. The second equality is by definition of $L_{\Lambda}$. The third equality is due to the Gröbner basis fact that for any ideal $I$ and any term order $\prec$, the image in $k[\mathbf{x}] / I$ of the standard monomials (those monomials not in $\left.i n_{\prec}(I)\right)$ form a $k$-basis of $k[\mathbf{x}] / I$. The fourth equality follows from (5.1).

The next two lemmas prove the isomorphism of the Tor-groups claimed in Proposition 5.4, by describing a relation between the minimal free resolutions of $k$ over $R$ and over $\operatorname{gr}_{\mathfrak{m}}(R)$; this relation is described by a filtration.

Let $G=\bigoplus_{i=1}^{n} R e_{i}$ be a free $R$-module of rank $n$. Define a standard filtration with shifts $a_{1}, \ldots, a_{n}$ by $F_{j} G=\bigoplus_{i=1}^{n} \mathfrak{m}^{j-a_{i}} e_{i}$. Then $\operatorname{gr}_{F}(G)=\bigoplus_{i=1}^{n} \operatorname{gr}_{\mathfrak{m}}(R)\left(-a_{i}\right)$.

Let $(G ., d$.) be a resolution of $k$ over $R$ and let $F$ be a standard filtration on $G$. . Assume $d_{j}$ is compatible with the filtration $F$, that is, $d_{j}\left(F_{i} G_{j}\right) \subset$ $F_{i} G_{j-1}$. Then there is an obvious induced graded differential

$$
\left.\operatorname{gr}_{F}\left(d_{j}\right)\right|_{F_{i} G_{j} / F_{i+1} G_{j}}: F_{i} G_{j} / F_{i+1} G_{j} \rightarrow F_{i} G_{j-1} / F_{i+1} G_{j-1}
$$


Clearly, $\operatorname{gr}_{F}\left(d_{j}\right) \circ \operatorname{gr}_{F}\left(d_{j+1}\right)=0$. Thus $\left(\operatorname{gr}_{F}(G\right.$. $\left.), \operatorname{gr}_{F}(d).\right)$ is a graded complex.

The following two lemmata can be proven by standard arguments from commutative algebra. We therefore leave the detailed proof to the reader.

Lemma 5.7. Let $(R, \mathfrak{m})$ be either local or $\mathbf{N}^{d}$-graded. There is a free (but not necessary minimal) resolution $(G ., d$.) of $k$ over $R$ and a standard filtration $F$ on $G$., such that $\left(\operatorname{gr}_{F}(G),. \operatorname{gr}_{F}(d).\right)$ is a minimal graded free resolution of $k$ over $\operatorname{gr}_{\mathfrak{m}}(R)$. If $R$ is $\mathbf{N}^{d}$-graded then $\left(\operatorname{gr}_{F}(G),. \operatorname{gr}_{F}(d).\right)$ is also.

As a consequence of this lemma, we conclude that when $(R, \mathfrak{m})$ is local we have

$$
\operatorname{dim}_{k} \operatorname{Tor}_{i}^{R}(k, k) \leq \operatorname{dim}_{k} \operatorname{Tor}_{i}^{\mathrm{gr}}(R)(k, k)
$$

for all $i$, and when $(R, \mathfrak{m})$ is $\mathbf{N}^{d}$-graded we have

$$
\operatorname{dim}_{k} \operatorname{Tor}_{i}^{R}(k, k)_{\lambda} \leq \operatorname{dim} \operatorname{Tor}_{i}^{\mathrm{gr}}(R)(k, k)_{\lambda}
$$

for any $i$ and any $\lambda \in \mathbf{N}^{d}$.

The natural filtration on $G$. with respect to $\mathfrak{m}$ is given by $F_{i} G_{j}=\mathfrak{m}^{i-j} G_{j}$, $j \leq i$ and $F_{i} G_{j}=G_{j}$ for $j>i$. In particular, $\operatorname{gr}_{\mathfrak{m}}\left(G_{j}\right)(-j) \cong \operatorname{gr}_{F}\left(G_{j}\right)=$ $\bigoplus_{i \geq 0} F_{i} G_{j} / F_{i+1} G_{j}$. Of course, the natural filtration is a standard filtration. Note, that the natural filtration of $G$. is a filtration of the complex $(G ., d$.) if and only if $d_{i}\left(G_{i}\right) \subseteq \mathfrak{m} G_{i-1}$.

Lemma 5.8. Let $(R, \mathfrak{m})$ be either local or $\mathbf{N}^{d}$-graded. Let $(G ., d$.) be a minimal free resolution of $k$ over $R$, and let $F$ be the natural filtration on $G$.. Then $R$ is Koszul if and only if $\left(\operatorname{gr}_{F}(G),. \operatorname{gr}_{F}(d).\right)$ is acyclic. If these equivalent conditions hold then $\left(\operatorname{gr}_{\mathfrak{m}}(G),. \operatorname{gr}_{\mathfrak{m}}(d).\right)$ is a minimal graded free resolution of $k$ over $\operatorname{gr}_{\mathfrak{m}}(R)$. Furthermore, if $R$ is a $\mathbf{N}^{d}$-graded $k$-algebra then $\left(\operatorname{gr}_{\mathfrak{m}}(G),. \operatorname{gr}_{\mathfrak{m}}(d).\right)$ is also multigraded.

As a consequence of this lemma, in the case when $(R, \mathfrak{m})$ is Koszul, the inequalities which followed from Lemma 5.7 can be sharpened to equalities: If $(R, \mathfrak{m})$ is Koszul and local we have

$$
\operatorname{dim}_{k} \operatorname{Tor}_{i}^{R}(k, k)=\operatorname{dim}_{k} \operatorname{Tor}_{i}^{\mathrm{gr}_{\mathrm{m}}(R)}(k, k)
$$

for all $i$, and if $(R, \mathfrak{m})$ is Koszul and $\mathbf{N}^{d}$-graded we have

$$
\operatorname{dim}_{k} \operatorname{Tor}_{i}^{R}(k, k)_{\lambda}=\operatorname{dim}_{k} \operatorname{Tor}_{i}^{\mathrm{gr}}(R)(k, k)_{\lambda}
$$

for all $i$ and all $\lambda \in \mathbf{N}^{d}$. Thus Proposition 5.4 follows. 


\section{Appendix: Combinatorial topology tools and lemmas.}

We collect here some of the tools from combinatorial topology that are used in Sections 3 and 4.

Let $X$ be a regular $\mathrm{CW}$-complex. By a covering $\mathcal{U}$ of $X$ we understand a finite collection $X_{1}, \ldots, X_{l}$ of closed subcomplexes of $X$ such that $X_{1} \cup \cdots \cup$ $X_{l}=X$. To each covering $\mathcal{U}$ of $X$ one associates its intersection poset $P^{\mathcal{U}}$. The intersection poset has elements $X_{J}=\bigcap_{j \in J} X_{j}$, for non-empty subsets $J$ of $\{1, \ldots, l\}$, and these are ordered by reversed inclusion - note that the poset is defined on the set of intersections $X_{J}$, so if $X_{J}=X_{J^{\prime}}$ for two different subsets $J, J^{\prime}$ of $\{1, \ldots, l\}$ then they contribute to a single element in the intersection poset. For an element $p$ in the intersection poset $P^{\mathcal{U}}$ we write $U_{p}$ to denote the intersection corresponding to $p$.

Recall, that the topological join $X * Y$ of two topological spaces $X, Y$ is defined as the quotient space

$$
X * Y:=X \times Y \times[0,1] /((x, 0) \sim x,(y, 1) \sim y) .
$$

Note that the join $\mathbf{S}^{0} * X$ of $X$ with a 0 -sphere is the same as the suspension $\operatorname{Susp}(X)$. The wedge $X \vee Y$ of two topological spaces $X$ and $Y$ is the space $(X \uplus Y) /(x \sim y)$ where two distinguished points $x \in X$ and $y \in Y$ are identified in the disjoint union of $X$ and $Y$. Note, if $X$ and $Y$ are pathconnected then the homotopy type of $X \vee Y$ does not depend on the choice of $x \in X$ and $y \in Y$.

We now formulate a version of the "Wedge Lemma" from $[\mathbf{Z Z}]$ (see also [WZZ]) that is adapted to our purposes.

Wedge Lemma 6.1 ([ZZ]). Let $\mathcal{U}$ be a covering of a regular $C W$-complex $X$ by closed subcomplexes $X_{1}, \ldots, X_{l}$. Let $P$ be the intersection poset of $\mathcal{U}$. Assume that for all $p \in P$ there is a point $c_{p} \in U_{p}$ such that all maps $U_{q} \rightarrow U_{p}$ are homotopic to the constant map to $\bigcup_{q>p} U_{q} \rightarrow\left\{c_{p}\right\}$. Then $X$ is homotopy equivalent to the wedge

$$
\bigvee_{p \in P} \Delta\left(P_{<p}\right) * U_{p}
$$

in which the wedge identifies the vertex $p$ in $\Delta\left(P_{<p}\right)$ with the vertex $p$ in $\Delta\left(P_{<\hat{1}}\right)$, where $\hat{1}$ is the top element of $P$ corresponding to the intersection $\bigcap_{i=1}^{l} X_{i}$.

For the proof of Lemma 3.3 we need the following lemma. In the formulation, $X \simeq Y$ means that the topological spaces $X$ and $Y$ are homotopy equivalent. 
Lemma 6.2. Let $X, Y$ be $C W$-complexes. If $X$ and $Y$ are l-connected and $X \cap Y$ is a sub-complex of dimension at most $l$, then $X \cup Y \simeq X \vee Y \vee$ $\operatorname{Susp}(X \cap Y)$. In particular, if

$$
X \simeq \bigvee_{i=1}^{r} \mathbf{S}^{d_{i}^{X}}, \quad Y \simeq \bigvee_{j=1}^{s} \mathbf{S}^{d_{j}^{Y}}, \quad X \cap Y \simeq \bigvee_{k=1}^{t} \mathbf{S}^{d_{k}^{X \cap Y}}
$$

and $\operatorname{dim}(X \cap Y)<d_{i}^{X}, d_{j}^{Y}$ for all $i, j$ then $X \cup Y$ is homotopy equivalent to the one-point wedge of spheres

$$
X \cup Y \simeq \bigvee_{i=1}^{r} \mathbf{S}^{d_{i}^{X}} \vee \bigvee_{j=1}^{s} \mathbf{S}^{d_{j}^{Y}} \vee \bigvee_{k=1}^{t} \mathbf{S}^{d_{k}^{X \cap Y}+1}
$$

Proof. Use the covering

$$
X \hookleftarrow X \cup Y \hookrightarrow Y
$$

of $X \cap Y$ and the Wedge Lemma 6.1. Observe that this lemma applies since both inclusions are null-homotopic, due to the fact that every map from a space of dimension at most $l$ to an $l$-connected space is null-homotopic. By the Wedge Lemma, the homotopy type of $X \cup Y$ is given by $X \vee Y \vee$ $\operatorname{Susp}(X \cap Y)$ since the order complex of the poset below $X \cap Y$ is just two points, i.e. the 0-sphere $\mathbf{S}^{0}$.

The next lemma is needed in the proof of Theorem 4.2.

Lemma 6.3. Let $\mathcal{A}$ be an arrangement of hyperplanes in $\mathbf{R}^{n}$ and $\mathbf{S}^{n-1}$ the unit sphere. Then the intersection $\mathbf{S}^{n-1} \cap \mathcal{A}$ has the homotopy of a wedge of $(n-2)$-spheres, with the number of spheres being one fewer than the number of chambers cut out by $\mathcal{A}$.

Proof. It is easily seen that intersection $\mathbf{S}^{n-1} \cap \mathcal{A}$ carries the structure of an $(n-2)$-dimensional regular CW-complex, and therefore its homology vanishes in dimensions greater than $n-2$. The quotient space $\mathbf{S}^{n-1} /\left(\mathbf{S}^{n-1} \cap\right.$ $\mathcal{A})$ is a one-point wedge of as many $(n-1)$-spheres as there are chambers cut out by $\mathcal{A}$ on $\mathbf{S}^{n-1}$. Thus $\widetilde{H}_{i}\left(\mathbf{S}^{n-1} /\left(\mathbf{S}^{n-1} \cap \mathcal{A}\right)\right)$ is free and concentrated in dimension $n-1$. By the long exact sequence of the pair $\left(\mathbf{S}^{n-1}, \mathbf{S}^{n-1} \cap \mathcal{A}\right)$, the homology of $\mathbf{S}^{n-1} \cap \mathcal{A}$ is free, concentrated in dimension $n-2$, and has rank one smaller than $H_{n-1}\left(\mathbf{S}^{n-1} /\left(\mathbf{S}^{n-1} \cap \mathcal{A}\right)\right)$. Thus by the Hurewicz and Whitehead Theorems, the intersection $\mathbf{S}^{n-1} \cap \mathcal{A}$ for $n \geq 4$ is simply connected and has the homotopy of a wedge of $(n-2)$-spheres, with the number of spheres one smaller than the number of chambers cut out by $\mathcal{A}$. 
As an alternative to the previous proof, one could consult the much more general formula by Ziegler \& Živaljević in $[\mathbf{Z Z}]$ on the homotopy type of $\mathbf{S}^{n-1} \cap \mathcal{A}$ for an arbitrary real subspace arrangement $\mathcal{A}$.

Our last topological lemma is needed in the proof of Lemma 3.5.

Lemma 6.4. Let $P$ be a d-dimensional polytope and $F$ a face on its boundary. Then the union of all maximal faces of $P$ not containing $F$ is a shellable ball $\mathbf{B}^{d-1}$.

Proof. Choose a shelling of $P$ which shells the maximal faces containing $F$ last, e.g. by picking a linear functional on the vertices of the polar dual $P^{*}$ which orders the vertices in the dual face $F^{*}$ last (see $[\mathbf{D K}]$ for a definition of shelling and why this choice is possible). Then in this shelling, the union of all facets not containing $F$ gets shelled along the way, and hence is a ball $\mathbf{B}^{d-1}$.

Acknowledgments. Vic Reiner was partially supported by a University of Minnesota McKnight-Land Grant Fellowship. Volkmar Welker was partially supported by the DFG through "Habilitationstipendium" We 1479/3 and by MSRI as a "General Member" at the 1996/97 MSRI program on Combinatorics. The authors would like to thank Irena Peeva for many useful observations (including Example 2.2 and Proposition 2.3) and careful readings and helpful edits of earlier versions of the paper.

\section{References}

[Ab] S.S. Abhyankar, Local rings of high embedding dimension, Amer. J. Math., 83 (1967), 1073-1077.

[BF] J. Backelin and R. Fröberg, Poincaré series of short Artinian rings, J. Algebra, 96 (1985), 495-498.

[BGS] A. Björner, A. Garsia and R. Stanley, An introduction to Cohen-Macaulay partially ordered sets, in 'Ordered sets' (Banff, Alberta 1981), NATO-Reidel/Dordrecht, Boston, (1982), 583-615.

[BK] A. K. Bousfield and D. M. Kan, Homotopy limits, completions and localizations, Lecture Notes in Mathematics, 304, Springer-Verlag, Berlin, 1972.

[BH1] W. Bruns and J. Herzog, Cohen-Macualay Rings, Cambridge University Press, 1993.

[BH2] , Semigroup rings and simplicial complexes, J. Pure Appl. Algebra, 122 (1997), 185-208.

[BHV] W. Bruns, J. Herzog and U. Vetter, Syzygies and walks, Commutative Algebra, World Scientific, (1994), 36-57.

[CF] P. Cartier and D. Foata, Problèmes combinatoire des commutations et réarrangements, Lecture Notes in Mathematics, 85, Springer, Heidelberg, 1969.

[CLO] D. Cox, J. Little and D. O'Shea, Ideals, varieties, and algorithms: An introduction to computational algebraic geometry and commutative algebra, Springer-Verlag, New York, 1997. 
[DK] G. Danaraj and V. Klee, Shellings of spheres and polytopes, Duke Math. J., 41 (1974), 443-451.

[Ei] D. Eisenbud, Commutative Algebra with a View Towards Algebraic Geometry, Springer Verlag, New York, 1995.

[Fr1] R. Fröberg, Determination of a class of Poincaré series, Math. Scand., 37 (1975), 29-39.

[Fr2] Connections between a local ring and its associated graded ring, J. Algebra, 111 (1987), 300-305.

[GKZ] I.M. Gelfand, M.M. Kapranov and A.V. Zelevinsky, Discriminants, Resultants and Multidimensional Determinants, Birkhäuser, Boston, 1994.

[GZ] C. Greene and T. Zaslavsky, On the interpretation of Whitney numbers through arrangements of hyperplanes, zonotopes, non-radon partitions, and orientations of graphs, Trans. Amer. Math. Soc., 280 (1983), 97-125.

[LS] O.A. Laudal and A. Sletsjøe, Betti numbers of monoid algebras. Applications to 2-dimensional torus embeddings, Math. Scand., 56 (1985), 145-162.

[Ma] S. Mac Lane, Homology, Springer-Verlag, 1975.

[OT] P. Orlik and H. Terao, Arrangements of Hyperplanes, Springer-Verlang, 1992.

[PRS] I. Peeva, V. Reiner and B. Sturmfels, How to shell a monoid, Math. Ann., 3b (1998), 379-393.

[Qu] D. Quillen, Homotopy properties of the poset of nontrivial p-subgroups of a group, Adv. in Math., 28(2) (1978), 101-128.

[Sa] J.D. Sally, On the associated graded ring of a local Cohen-Macaulay ring, J. Math. Kyoto U., 17 (1977), 19-21.

[Sta] R.P. Stanley, Enumerative Combinatorics, I, Wadsworth \& Brooks/Cole, Monterey, CA, 1986.

[Wa] M. Wachs, Whitney homology of semipure shellable posets, preprint, July 1996.

[WZZ] V. Welker, G.M. Ziegler and R. Živaljević, Comparison lemmas and applications for diagrams of spaces, submitted, preprint, 1995.

[ZZ] G.M. Ziegler and R. Živaljević, Homotopy types of subspace arrangements via diagrams of spaces, Math. Ann., 295 (1993), 527-548.

Received April 17, 1997 and revised August 20, 1997.

UNIVERSITÄT GH-ESSEN

45117 Essen, Germany

E-mail address: math300@uni-essen.de

UNIVERSITY OF MinNESOTA

MinneAPOLIS, MN 55455

E-mail address: reiner@math.umn.edu

TU-BERLIN

10623 Berlin, Germany

E-mail address: welker@math.tu-berlin.de 